\title{
Incidence, clinical management, and mortality risk following self harm among children and adolescents: cohort study in primary care
}

\author{
Catharine Morgan, ${ }^{1}$ Roger T Webb, ${ }^{2}$ Matthew J Carr, ${ }^{2}$ Evangelos Kontopantelis, ${ }^{3}$ Jonathan \\ Green, ${ }^{4}$ Carolyn A Chew-Graham, ${ }^{5}$ Nav Kapur, ${ }^{6}$ Darren M Ashcroft ${ }^{1}$
}

For numbered affiliations see end of article.

Correspondence to: CMorgan Cathy.Morgan@manchester.ac.uk Additional material is published online only. To view please visit the journal online.

Cite this as: $B M J$ 2017;359:j4351 http://dx.doi.org/10.1136/bmj.j4351

Accepted: 11 September 2017

\section{ABSTRACT}

OBJECTIVES

To examine temporal trends in sex and age specific incidence of self harm in children and adolescents, clinical management patterns, and risk of cause specific mortality following an index self harm episode at a young age.

DESIGN

Population based cohort study.

SETTING

UK Clinical Practice Research Datalink-electronic health records from 674 general practices, with practice level deprivation measured ecologically using the index of multiple deprivation. Patients from eligible English practices were linked to hospital episode statistics (HES) and Office for National Statistics (ONS) mortality records.

PARTICIPANTS

For the descriptive analytical phases we examined data pertaining to 16912 patients aged 10-19 who harmed themselves during 2001-14. For analysis of cause specific mortality following self harm, 8638 patients eligible for HES and ONS linkage were matched by age, sex, and general practice with up to 20 unaffected children and adolescents $(n=170274)$.

\section{WHAT IS ALREADY KNOWN ON THIS TOPIC}

Self harm is the strongest risk factor for subsequent suicide, with suicide being the second commonest cause of death before age 25 worldwide

Increases in child and adolescent suicide rates have been identified in recent years, and psychological distress at young age has also been reported as increasing sharply, but the incidence of non-fatal self harm is difficult to quantify at population level in the absence of national data sources

A gap exists in the evidence base for temporal trends relating to self harm among children and adolescents, with observation periods of published studies currently extending no further than 2012, and only sparse evidence available from primary care populations

\section{WHAT THIS STUDY ADDS}

A $68 \%$ increase in self harm incidence was found among girls aged 13-16 between 2011 and 2014, which indicates an urgent need to develop and implement effective interventions for girls in their early-mid teens

The likelihood of referral was lowest in practices in the most deprived localities where incidence of self harm was highest-an illustration of the "inverse care law," whereby quantity or quality of healthcare service provision is inversely associated with the level of healthcare need

The high relative risks observed for cause specific premature mortality, and for suicide and fatal acute alcohol or drug poisonings in particular, highlight the importance of effective interagency collaboration to enhance safety and future mental wellbeing for distressed young people

\section{MAIN OUTCOME MEASURES}

In the first phase, temporal trends in sex and age specific annual incidence were examined. In the second phase, clinical management was assessed according to the likelihood of referral to mental health services and psychotropic drug prescribing. In the third phase, relative risks of all cause mortality, unnatural death (including suicide and accidental death), and fatal acute alcohol or drug poisoning were estimated as hazard ratios derived from stratified Cox proportional hazards models for the self harm cohort versus the matched unaffected comparison cohort.

\section{RESULTS}

The annual incidence of self harm was observed to increase in girls (37.4 per 10000 ) compared with boys (12.3 per 10000$)$, and a sharp $68 \%$ increase occurred among girls aged 13-16, from 45.9 per 10000 in 2011 to 77.0 per 10000 in 2014 . Referrals within 12 months of the index self harm episode were $23 \%$ less likely for young patients registered at the most socially deprived practices, even though incidences were considerably higher in these localities. Children and adolescents who harmed themselves were approximately nine times more likely to die unnaturally during follow-up, with especially noticeable increases in risks of suicide (deprivation adjusted hazard ratio $17.5,95 \%$ confidence interval 7.6 to 40.5 ) and fatal acute alcohol or drug poisoning $(34.3,10.2$ to 115.7$)$.

\section{CONCLUSIONS}

Gaining a better understanding of the mechanisms responsible for the recent apparent increase in the incidence of self harm among early-mid teenage girls, and coordinated initiatives to tackle health inequalities in the provision of services to distressed children and adolescents, represent urgent priorities for multiple public agencies.

\section{Introduction}

Self harm in children and adolescents is a major public health problem in many countries. In a large population based cohort study from Australia, for example, $8 \%$ of adolescents aged less than 20 years reported harming themselves at some time. ${ }^{1}$ Self harm is highly correlated with the presence of anxiety disorders and depression, ${ }^{1}{ }^{2}$ with prolonged poor psychological health in childhood linked to the onset of common mental illnesses in adulthood. ${ }^{3}$ Non-fatal self harm is also the strongest risk factor for subsequent suicide, ${ }^{4}$ which is the second most common cause of death among 10-24 year olds worldwide after road traffic incidents. ${ }^{5}$ Suicide rates vary considerably globally. ${ }^{6}$ In 
the United Kingdom, suicide rates among adolescents aged 15-19 years have increased from 3.2 to 5.4 per 100000 between 2010 and 2015, respectively. ${ }^{7}$ Half of adolescents who die by suicide have a history of self harm. ${ }^{8}$ In addition, there has been an increase in reports of mental health problems, including self harm, from teachers ${ }^{9}$ and from charity based or third sector organisations ${ }^{10}$ in frontline contact with children and adolescents. In the UK, the national suicide prevention strategy $^{11}$ recently expanded its scope by aiming to reduce self harm rates as a common precursor to suicide.

However, to achieve this goal the frequency and course of self harm in children and adolescents at population level must first be accurately quantified. The elusive nature of self harm represents a major obstacle. Less than a quarter of children and adolescents who self harm are believed to present to healthcare services. ${ }^{12}$ Current figures, based largely on hospital data, ${ }^{13}$ likely underestimate the incidence of self harm. People who self harm and present to general practitioners alone are not captured and only half of self harm presentations to secondary care result in hospital admissions. ${ }^{14} 15$ In the healthcare systems of most developed countries, primary care is often the first point of contact for mental health problems. In the UK, primary care electronic medical records offer the opportunity to identify a large cohort of children and adolescents who have harmed themselves, allowing comprehensive reporting over time and supporting advanced analyses, including linkage to mortality data from the Office for National Statistics (ONS).

Utilising the Clinical Practice Research Datalink (CPRD), one of the world's largest databases of electronic primary care patient records, we aimed: to investigate temporal trends in the incidence of self harm during 2001-14 among children and adolescents aged 10-19 years; to assess referral rates to specialist mental health services and prescribing of psychotropic drugs in the year after the index self harm episode; and to examine the risks of all cause and cause specific mortality among children and adolescents following self harm compared with unaffected peers of the same age and sex.

\section{Methods}

Data source

The CPRD is a UK wide anonymised primary care patient records database, capturing general practitioner consultations and recording symptoms, diagnoses, prescribed drugs, and referrals to secondary care services. The CPRD contains over 4.4 million active patient records from 674 registered general practices in the four nations of the UK: England, Northern Ireland, Scotland, and Wales. It covers 6.9\% of the UK population and is broadly representative of the national population in terms of age, sex, and ethnicity. ${ }^{16}$ Approximately $60 \%$ of practices, all of which are located in England, participate in the CPRD linkage scheme, which facilitates routine linkage to Office for National Statistics (ONS) mortality records, to hospital episode statistics (HES), and to the index of multiple deprivation. ${ }^{17}$ This deprivation measure is derived from a combination of several socioeconomic indicators for small areas based on practices' localities or patients' residential postcodes. It is ranked within each UK country, and then placed in fifths for least to most deprived areas.

\section{Study design and population}

Children and adolescents aged 10-19 years and with a self harm record during 2001 to 2014 were included in this cohort study. Self harm was defined using the UK National Institute for Health and Care Excellence clinical guidelines as "any act of self-poisoning or self-injury, irrespective of motivation." ${ }^{18}$ Non-suicidal self injury or suicide attempt are classifications used in some settings but are reliant on establishing the intent of the behaviour, which is difficult to discern in children and adolescents. Two authors (CAC-G and NK) identified self harm records across a broad spectrum of codes from milder forms of non-suicidal behaviour to near-fatal suicide, with each code subject to rigorous clinical review. ${ }^{19}$ A previously published validation study expressed caution about ascertainment of death by suicide, using Read codes in CPRD, and thus the authors emphasised the need for linkage to national mortality records. ${ }^{20}$ This study found no such concerns in relation to ascertaining cases of non-fatal self harm using Read coding. Figure 1 outlines the numbers of patient records included at each analytical phase.

\section{Statistical analysis}

In the first phase of analysis, we calculated annual incidence from the number of children and adolescents presenting with a self harm episode during each calendar year in relation to the total number of children and adolescents at risk during the same year. Having stratified on age band, index of multiple deprivation, and region of residence, we then applied stratum specific rates to calculate directly standardised incidence rates. Age was categorised as pre-teenager (10-12), early teenager (13-16), and late teenager (17-19) year bands, and the index of multiple deprivation was placed into fifths based on general practice location.

In the second analytical phase, clinical management was assessed according to referrals to specialist mental health services and prescribing of psychotropic drugs in the first year after the index self harm episode. Psychiatric referrals were captured using the Family Health Services Authority (FHSA) "Psychiatry" code, National Health Service specialty fields, and relevant Read codes often used during consultations. Psychotropic drugs included prescriptions for antidepressants, antipsychotics, and hypnotics or anxiolytics. We stratified clinical management variables by sex, age group, and practice level deprivation fifth. Mental illness comorbidity was examined according to diagnoses of depression, anxiety disorders, attentiondeficit/hyperactivity disorder (ADHD), eating disorders, personality disorders, and autism spectrum 
Phase 1: Characteristics, incidence, and temporal trends Self harm in children and adolescents $(n=16912)$ Girls ( $n=12398 ; 73.3 \%$ ); boys ( $n=4514 ; 26.7 \%$ )

Median age 16 (interquartile range $15-18$ ) years

Deprivation fifth $1(\mathrm{n}=2537 ; 15.0 \%)$ least

$2(n=2992 ; 17.7 \%)$

$3(n=3395 ; 20.1 \%)$

$4(n=3937 ; 23.3 \%)$

$5(n=4051 ; 24.0 \%)$ most

Inclusion criteria:

Age $10-19$ years

First recorded self harm episode between 1 January 2001 and 31 December 2014

UK wide practice and an individuals' data meeting quality "up to standard" CPRD criteria

Registered with practice for 12 months before index self harm episode
Excluded self harm episodes after 31 December $2013(n=1411)$

Children and adolescents with self harm and potential 1 year follow-up $(n=15501)$

\section{Excluded $(n=1973)$ :}

Death $(n=50)$

Transfer out of practice $(n=1278)$

Practice no longer contributing during

12 month follow-up $(n=645)$

Phase 2: Clinical management

Children and adolescents with self harm

$(n=13528)$

Girls ( $n=9883 ; 73.1 \%)$; boys ( $n=3645 ; 26.9 \%)$ following self harm
Children and adolescent with self harm recorded up to 31 March 2014

English practices in which patients were eligible for linkage to hospital and mortality records

- Comparison group had no self harm record up to index date of matched self harm

Adolescent with self harm matched by age, sex, and practice with up to 20 comparators

$\downarrow$

\section{Phase 3: Cause specific mortality} risks following self harm Children and adolescents with self harm $(n=8638)$

Girls ( $n=6383 ; 73.9 \%)$; boys $(n=2255 ; 26.1 \%)$ Median age 16 (interquartile range $15-18$ ) years Deprivation fifth 1 ( $n=1460 ; 16.9 \%)$ least $2(n=1498 ; 17.4 \%)$ $3(n=1596 ; 18.5 \%)$ $4(\mathrm{n}=1929 ; 22.4 \%)$ $5(n=2149 ; 24.8 \%)$ most

Comparison group of children and adolescent $(n=170274)$

Girls ( $n=125472 ; 73.7 \%)$

Boys $(n=44802 ; 26.3 \%)$

\section{Fig 1 | Derivation of each cohort across the study's three analytical phases}

disorders, conduct disorder, schizophrenia spectrum disorders, obsessive compulsive disorder, bipolar disorder, tics, and social dysfunction.

For the third and final analytical phase, we implemented a matched cohort design to investigate cause specific mortality risk. We restricted the study cohort for this phase to include only patients registered at English CPRD practices participating in the HES and ONS linkage scheme. Incident self harm episodes recorded in the CPRD before 31 March 2014 were included, where no previous self harm episode was identified from either the primary care record or the linked HES data record. Self harm episodes in the HES data were delineated using ICD-10 (international classification of disease, 10th revision) ${ }^{21}$ codes X60-X84.9. We matched each person with an incident episode by age, sex, and registered practice with up to 20 comparison individuals with no record of self harm by the index self harm date. This large comparison group maximised statistical power and precision (fig 1).

To classify cause specific mortality following self harm, we categorised the underlying cause of death code according to ICD-10: all cause mortality, all natural deaths; all unnatural deaths and suicide (including and excluding open verdicts); accident; and acute alcohol or drug poisoning. To estimate risks of all cause and cause specific mortality, we performed Cox regression analysis stratified by matched set, producing both unadjusted hazard ratios and those adjusted for deprivation fifth by patient postcode. The index date in the self harm and matched comparison cohorts was defined when the first recorded self harm event occurred. We right censored the records at the end of the follow up period (31 March 2014), the practice last data collection date, date of transfer to another practice, or death, whichever date was earliest. Evidence for differences in sex specific hazard ratios was assessed using likelihood ratio interaction tests. The proportional hazards assumption was assessed using Schöenfeld's residuals, ${ }^{22}$ with no evidence of this being violated in any of the fitted models.

All relevant code lists are published online (www. clinicalcodes.org $)^{23}$ and analyses were performed using Stata/SE 14.2.

\section{Results}

Phase 1: characteristics of the self harm cohort, incidence, and temporal trends

During the 2001-14 observation period, 16912 children and adolescents were identified with at least one episode of self harm, of whom 12398 (73.3\%) were girls and $4514(26.7 \%)$ boys. Relatively high prevalence values for depression and anxiety disorders were observed in the self harm cohort, with depression diagnoses recorded in over a third of girls and in over a quarter of boys. ADHD, autism spectrum disorders, conduct disorder, and schizophrenia spectrum disorders were more commonly seen in boys than in girls and eating disorders more prevalent in girls than in boys (see supplementary appendix 1). Of the 13980 $(82.7 \%)$ index episodes with method of self harm recorded, $84.1 \%$ were coded as drug overdoses, with an additional $2.5 \%$ identified as other self poisonings. A further $12.3 \%$ were self cutting episodes, and the remaining $1.1 \%$ involved various methods, including hanging, suffocation, jumping, and scalding. Within 12 months of the index episode, repeat self harm was common $(\mathrm{n}=3628,21.5 \%)$; more among girls $(22.6 \%)$ than among boys $\left(18.3 \% ; \chi^{2}=35.3\right)$; $\mathrm{P}<0.001$. Among those who had records of repeat self harm, 2685 (74.0\%) had one repeat episode recorded, 641 $(17.7 \%)$ had two repeat episodes, and 302 (8.3\%) had three or more further episodes within 12 months of their index episode.

Considerably higher standardised annual self harm incidence rates were observed in girls 37.4 (95\% confidence interval 36.8 to 38.1) per 10000 compared with boys 12.3 (11.9 to 12.6). Among children and adolescents registered with practices in the most deprived areas, the annual incidence per 10000 was increased $(27.1,95 \%$ confidence interval 26.1 to 28.2) compared with those consulting practices in the least deprived areas (19.6, 18.6 to 20.6). Age specific incidence rates per 10000 showed a noticeable increase across the study's observation period among 


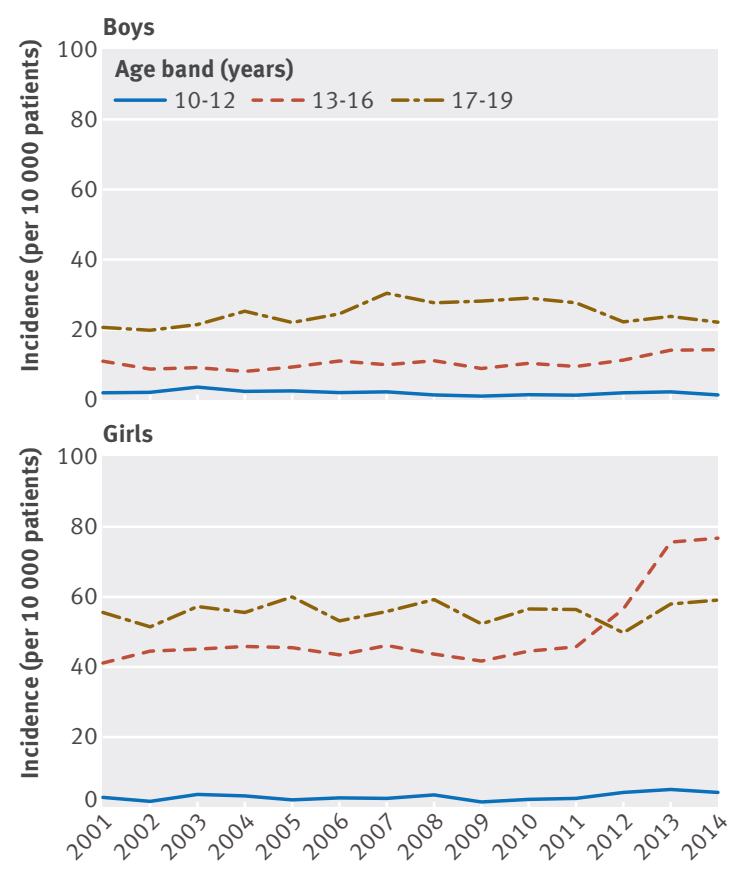

Year

Fig 2 | Temporal trends in annual age specific self harm incidence stratified by sex. Standardised by index of multiple deprivation fifth and region of residence

girls aged 13-16, increasing by 68\%; from 45.9 (95\% confidence interval 41.7 to 50.0) in 2011 to 76.9 (70.7 to 83.2) in 2014 (fig 2). For 2012, 2013, and 2014, the incidence among girls aged 13-16 was higher than among those aged 17-19 years. Thus, from a lower baseline the incidence among females in their earlymid teenage years advanced and surpassed that seen in older female teenagers. The sharp increase was specific to girls aged 13-16 years, as no noticeable increases in incidence over time were seen in females aged $10-12$ or $17-19$, or among males in any of the three age stratums examined.

\section{Phase 2: clinical management during 12 months following self harm}

No referral to mental health services was documented for 7552 (55.8\%) children and adolescents. However, 1676 (12.4\%) were referred before the index self harm episode and a further 1905 (14.1\%) were referred more than 12 months afterwards. Of the 2395 (17.7\%) children and adolescents referred within 12 months, a higher percentage of girls were referred than boys (18.2\% v 16.5\%; $\chi^{2}=5.06 ; \mathrm{P}=0.02$, table 1 ). Children and adolescents from general practices located in the most deprived areas were $23 \%$ less likely to be referred in the first 12 months after an index self harm episode compared with those practices in the least deprived areas (hazard ratio 0.77, 95\% confidence interval 0.67 to $0.89 ; \mathrm{P}=0.001)$. In addition, girls consulting at practices in the most deprived localities were $19 \%$ less likely to receive a prescription for a psychotropic in the first 12 months compared with the least deprived localities $(0.81,0.71$ to $0.92 ; \mathrm{P}=0.002)$. Overall, more than a fifth of children and adolescents were prescribed antidepressants, with more girls prescribed them than boys $\left(22.9 \%\right.$ v $20.4 \% ; \chi^{2}=9.7$; $\mathrm{P}=0.002$, table 2). Conversely, boys were more likely to be prescribed hypnotics or anxiolytics $(8.3 \%$ V 5.8\%;

\begin{tabular}{|c|c|c|c|c|c|}
\hline \multirow{2}{*}{ Subgroups } & \multirow[b]{2}{*}{ Total No } & \multicolumn{2}{|c|}{ Referred to mental health services } & \multicolumn{2}{|c|}{ Psychotropic drugs* } \\
\hline & & No (\%) & $x^{2}$ testt & No $(\%)$ & $x^{2}$ testt \\
\hline Overall & 13528 & $2395(17.7)$ & & $3514(26.0)$ & \\
\hline Boys & 3645 & $601(16.5)$ & & $949(26.0)$ & \\
\hline \multicolumn{6}{|l|}{ Age (years): } \\
\hline $10-12$ & 192 & $27(14.1)$ & \multirow[t]{3}{*}{$x^{2}=1.8 ; P=0.18$} & $15(7.8)$ & \multirow{3}{*}{$x^{2}=124.0 ; P=0.0001$} \\
\hline $13-16$ & 1260 & $230(18.3)$ & & $223(17.7)$ & \\
\hline 17-19 & 2193 & $344(15.7)$ & & $711(32.4)$ & \\
\hline \multicolumn{6}{|l|}{ Deprivation fifths: } \\
\hline 1 (least deprived) & 513 & $98(19.1)$ & \multirow[t]{6}{*}{$x^{2}=8.5 ; P=0.004$} & $134(26.1)$ & \multirow{5}{*}{$x^{2}=0.3 ; P=0.56$} \\
\hline 2 & 584 & $110(18.8)$ & & $153(26.2)$ & \\
\hline 3 & 709 & $115(16.2)$ & & $192(27.1)$ & \\
\hline 4 & 913 & $149(16.3)$ & & $237(26.0)$ & \\
\hline 5 (most deprived) & 926 & $129(13.9)$ & & $233(25.2)$ & \\
\hline Girls & 9883 & $1794(18.2)$ & & $2565(26.0)$ & \\
\hline \multicolumn{6}{|l|}{ Age (years): } \\
\hline $10-12$ & 258 & $46(17.8)$ & \multirow[t]{3}{*}{$x^{2}=1.6 ; P=0.20$} & $18(7.0)$ & \multirow{3}{*}{$x^{2}=777.6 ; P=0.0001$} \\
\hline $13-16$ & 5469 & 969 (17.2) & & $871(15.9)$ & \\
\hline 17-19 & 4156 & 779 (18.7) & & $1676(40.3)$ & \\
\hline \multicolumn{6}{|l|}{ Deprivation fifths: } \\
\hline 1 (least deprived) & 1526 & $321(21.0)$ & \multirow[t]{5}{*}{$x^{2}=35.3 ; P=0.0001$} & $417(27.3)$ & \multirow{5}{*}{$x^{2}=8.8 ; P=0.003$} \\
\hline 2 & 1828 & $384(21.0)$ & & $518(28.3)$ & \\
\hline 3 & 1940 & $359(18.5)$ & & $489(25.2)$ & \\
\hline 4 & 2268 & $380(16.8)$ & & $582(25.7)$ & \\
\hline 5 (most deprived) & 2321 & $350(15.1)$ & & $559(24.1)$ & \\
\hline
\end{tabular}

*Including: antidepressants, antipsychotics, and hypnotics or anxiolytics.

tKruskal-Wallis test. 


\begin{tabular}{|c|c|c|c|c|}
\hline Psychotropic drugs & All $(n=13528)$ & Boys $(n=3645)$ & Girls $(n=9883)$ & $x^{2}$ test \\
\hline Serotonin reuptake inhibitors & $2610(86.9)$ & $621(83.6)$ & $1989(87.9)$ & \\
\hline Tricyclic antidepressants & $186(6.2)$ & $46(6.2)$ & $140(6.2)$ & \\
\hline Other antidepressants & $209(7.0)$ & $76(10.2)$ & $133(5.9)$ & \\
\hline First generation & $208(37.5)$ & $42(20.6)$ & $166(47.3)$ & \\
\hline Second generation & $346(62.3)$ & $161(78.9)$ & $185(52.7)$ & \\
\hline Hypnotics or anxiolytics & $872(6.5)$ & $302(8.3)$ & $570(5.8)$ & $x^{2}=28.0 ; P<0.001$ \\
\hline
\end{tabular}

$\left.\chi^{2}=28.0 ; \mathrm{P}<0.001\right)$ or antipsychotics (5.6 $v 3.6 \%$; $\chi^{2}=28.3$; $\left.\mathrm{P}<0.001\right)$ than girls during the first year after self harm. In both sexes, a higher proportion of prescriptions was evident for second generation than first generation antipsychotics, with the proportion of second generation prescriptions for boys considerably higher than that for girls.

\section{Phase 3: cause specific mortality risks following} self harm

Overall, 8638 children and adolescents were in the self harm cohort and 170274 in the matched comparison cohort; $74 \%$ were girls and $26 \%$ boys. A total of 43 deaths occurred among young people in the self harm cohort and 176 in the comparison cohort, of which $65.1 \%$ (self harm cohort) compared with 26.6\% (comparison cohort) were classified as unnatural deaths. Children and adolescents who self harmed were an estimated nine times more likely to die unnaturally during the follow-up period than their unaffected peers (deprivation adjusted hazard ratio 9.35, 95\% confidence interval 5.84 to 14.97 ). In the self harm cohort the risk was increased for all cause mortality and for each cause of death category examined, with especially noticeable increases in risk observed for suicide $(17.48,7.55$ to 40.46) and for fatal acute alcohol or drug poisoning (34.33, 10.19 to 115.69) (table 3). Boys in the self harm cohort had a higher increase in risk of all cause mortality compared with their unaffected male peers and also compared with the equivalent sex specific relative risk among girls (likelihood ratio: $\chi^{2}=5.0 ; \mathrm{P}=0.03$ ). Across all mortality categories examined, the deprivation adjusted hazard ratios were of a similar magnitude to the unadjusted estimates.

\section{Discussion}

In this cohort study we found a high incidence of self harm and statistically significant differences in incidence between boys and girls, with an apparent $68 \%$ increase in rates among girls aged $13-16$ years between 2011 and 2014. A lower likelihood of referral to specialist mental health services was observed at practices in more socially deprived localities, although the incidence of self harm was increased in these poorer practice populations. Following the initial self harm episode, the frequency of repeat self harm was high in both girls and boys. Children and adolescents were at noticeably increased risk of dying at a young age following self harm compared with their peers of the same age and sex without a history of self harm, particularly by suicide and acute alcohol or drug poisoning.

\section{Incidence of self harm}

We report an increased annual incidence of self harm in girls (37.4 per 10000) compared with boys (12.3 per 10000). This is consistent with previous hospital based studies ${ }^{1324}$ and a recent primary care based study reporting on self poisoning. ${ }^{25}$ Lower annual incidence rates were reported from a study based in six hospitals, 30.2 (95\% confidence interval 26.9 to 33.5 ) per 10000 for girls aged 10-14 years and 6.7 (5.2 to 8.2) per 10000 for boys, ${ }^{13}$ although caution is required when comparing rates across studies, as differences in

\begin{tabular}{|c|c|c|c|c|c|c|}
\hline \multirow[b]{2}{*}{ Cause of death* } & \multicolumn{2}{|c|}{ Self harm cohort $(n=8638)$} & \multicolumn{2}{|c|}{$\begin{array}{l}\text { Comparison cohort } \\
(n=170274)\end{array}$} & \multicolumn{2}{|l|}{ Hazard ratio $(95 \% \mathrm{Cl})$} \\
\hline & No of deaths & $\begin{array}{l}\text { Rate per } 1000 \\
\text { person years }\end{array}$ & $\begin{array}{l}\text { No of } \\
\text { deaths }\end{array}$ & $\begin{array}{l}\text { Rate per } 1000 \\
\text { person years }\end{array}$ & Unadjusted & Adjustedt \\
\hline All causes: & 43 & 5.47 & 176 & 1.11 & $5.78(4.08$ to 8.20$)$ & $5.71(4.02$ to 8.11$)$ \\
\hline Boys & 25 & 12.03 & 74 & 1.76 & $8.61(5.31$ to 13.95$)$ & $8.64(5.29$ to 14.12$)$ \\
\hline Girls & 18 & 3.11 & 102 & 0.88 & $3.95(2.35$ to 6.63$)$ & $3.93(2.33$ to 6.62$)$ \\
\hline All natural causes & 15 & 1.91 & 104 & 0.66 & $3.38(1.93$ to 5.90$)$ & $3.41(1.95$ to 5.99$)$ \\
\hline All unnatural causes & 28 & 3.56 & 72 & 0.45 & $9.31(5.85$ to 14.81$)$ & $9.35(5.84$ to 14.97$)$ \\
\hline Suicide & 13 & 1.65 & 17 & 0.11 & $18.67(8.32$ to 41.87$)$ & $17.48(7.55$ to 40.46$)$ \\
\hline Suicide (including open verdicts) & 16 & 2.04 & 24 & 0.15 & $17.31(8.52$ to 35.16$)$ & 16.95 (8.28 to 34.68$)$ \\
\hline Acute alcohol or drug poisoning & 11 & 1.40 & 6 & 0.04 & $38.20(13.23$ to 110.28$)$ & 34.33 (10.19 to 115.69$)$ \\
\hline Accident & 12 & 1.52 & 47 & 0.30 & $5.96(3.08$ to 11.53$)$ & $5.97(3.05$ to 11.68$)$ \\
\hline
\end{tabular}

*Cause of death categories are not mutually exclusive.

tAdjusted by index of multiple deprivation fifth. 
self harm definitions, derivation of rates, age ranges and age categorisations, and timescale used must be carefully considered.

Temporal trends in sex and age specific incidence In girls aged 13-16 years, there was a noticeable increase in the incidence of self harm over time, specifically during 2011-14. This might be an artefact caused by ascertainment anomalies occurring over this period, but this seems an unlikely explanation given that little change took place in incidence observed for the other female age groups, or for any of the three male age groups examined. It therefore seems reasonable to assume no differential misreporting over time. Previous published reports have also suggested an upward trend, although only up to 2012, with few specifically focusing on younger age categories. ${ }^{25-27} \mathrm{~A}$ threefold increased rate of probable and deliberate self harm was reported in females aged 10-19 years from 1994 to 2011 in a Danish hospital registry study. ${ }^{27}$ Another UK based study of primary care data from The Health Improvement Network (THIN) found that the intentional incidence of poisoning in females aged 15-17 years increased from 39.1 to 46.6 per 10000 person years between the two successive calendar periods, $1992-96$ to $2007-12 .{ }^{25}$ Recent data from the adult psychiatric morbidity survey in England, ${ }^{28}$ based on clinical interviews, indicate that $11.7 \%$ of $16-24$ year old females in 2007 reported ever self harming, increasing to $19.7 \%$ in 2014 . In a multicentre hospital based study in England, ${ }^{26}$ age standardised incidence of self harm appeared to decline from 2000 until 2009, with a possible increase and stabilising of rates from 2009 to 2012, although figures were based on a combined age range of 15-24 years. The trends reported in our study are consistent with the observed increase in psychological distress among adolescent girls $^{29}$ and the increase in suicide rates in England in females aged 10-29 years, of 2.7 per 100000 in 2012 to 3.2 per 100000 in $2015 .^{7}$

The high incidence of self harm among this age group is potentially due to the emergence of common mental health problems in females at this age and biological factors such as puberty and onset of sexual activity. ${ }^{30}$ Reasons for the recent temporal increase that we observed, however, are less obvious and are therefore speculative. Some evidence indicates that common mental disorders are becoming more common among this age group-perhaps a reflection that today's early adolescents are living in more stressful times. In a longitudinal study of young people in England, psychological distress was reported to have increased noticeably among girls aged 14 years compared with boys of the same age. ${ }^{29}$ Exposure to digital media and its potential impact on children and adolescents' mental health is the centre of continued media debate. Such technologies can be helpful and facilitate access to care but there is also a suggestion that extreme "connectedness" could have detrimental effects. $^{3132}$ It is possible that marked sex differences in online interactions play an important role. Early-mid teenage girls may become likely to engage in more communicative or socialising online in recent years, thereby becoming exposed to content that encourages or normalises self harm as a reaction to stressful events. In a recent report of self reported child wellbeing, it was noted that from 2009 to 2014 there was most striking dissatisfaction with self appearance ${ }^{33}$ among girls aged more than 12 years, with dissatisfaction increasing twofold from 2008 to 2013-14. England was reported to have the largest sex gap in children's satisfaction on this issue according to the children's world survey of more than 15 countries. ${ }^{34}$ It is also possible, however, that the increased incidence among early-mid teenage girls may be indicative of differential surveillance bias, with frontline services perhaps more likely to be alert to and to inquire about self harming behaviour in girls than in boys in this age range. Girls aged 13-16 may also be more likely to consult with a doctor than boys at these ages. The most plausible explanation could be that the marked increase in incidence observed reflects both a true rise in incidence together with more frequent help seeking behaviour and, if so, it is important that this need is met.

Clinical management 12 months following self harm In the first 12 months after an initial self harm episode, a large proportion of prescriptions for antidepressants was observed. Since there is a high prevalence of depressive symptoms in children and adolescents who self harm, antidepressants are the likely candidate for pharmacotherapy. This was substantiated by a recent CPRD study of children where increases in the past five years of prescription related records for depression were noted particularly among girls aged $15-17 .{ }^{35}$ The higher proportion of second generation antipsychotic drugs prescribed in boys compared with girls cannot be explained on the basis of our data alone. One possible explanation may relate to the different psychopathological profiles we found between boys and girls (see supplementary appendix 1). A higher prevalence of ADHD, conduct disorder, and schizophrenia spectrum disorders was found in boys and a higher prevalence of depression, eating disorders, and anxiety disorders in girls.

Referral rates to psychiatric services after self harm were low in our study, suggestive of less severe cases or possible reflection of the challenges in accessing specialist services in a timely manner. ${ }^{36}$ Furthermore, recent evidence suggests long waiting lists and high thresholds in accessing mental health services, with varying eligibility and exclusion criteria between geographical areas adding to these obstacles. ${ }^{37}$ The likelihood of referral was lowest in the most deprived localities where incidence of self harm was highest. This provides a stark illustration of Tudor Hart's "inverse care law," whereby quantity or quality of healthcare service provision is inversely associated with the level or severity of healthcare need in the population. $^{38}$

Over a quarter of children and adolescents in the cohort were treated with psychotropic drugs. Since self 
harm is a behaviour rather than a diagnosis, it is more likely the drug was prescribed to treat an underlying mental disorder rather than specifically for self harm. To date there have been no pharmacotherapy trials on the optimum treatment for children and adolescents who self harm. ${ }^{39}$ NICE guidelines refer to potential adverse drug reactions to antidepressants, including suicidal behaviour, self harm, and hostility, and therefore close monitoring is important, especially during the early treatment period..$^{40}$ Referring to NICE guidelines on short term management of children who self harm, psychological therapy should be offered as a first line treatment. ${ }^{18}$ The evidence base for the effectiveness of individual psychosocial therapy interventions is limited; however, in a recent large Danish register study, Erlangsen and colleagues found that provision of a psychosocial intervention of some kind resulted in lower risk of repeat self harm and all cause mortality at follow-up. ${ }^{41}$ Risk reduction was higher in young people and females, the group we identify here as showing increasing rates. A recent Cochrane review of self harm treatment specific to children and adolescents found 11 psychological intervention studies, although these were limited to single trials that were graded as being of low quality evidence. One of these, using dialectical behavioural therapy adapted for adolescents, suggested a reduction in the frequency of repeat self harm over time. ${ }^{39}$ Schools may also provide the opportunity for safe, familiar, and opportunistic monitoring of children. In a recent multicentre cluster randomised controlled trial across 10 European Union countries, a youth aware mental health programme intervention was shown to statistically significantly reduce self harm and suicidal ideation at 12 months. ${ }^{42}$ Families and carers are also likely to play an important role and, with increasing awareness of the negative impact that self harm can have on a young person's wider social circle, it is important that families and carers have the necessary tools and understanding to support their children while also feelingsupported themselves. Online resources such as healthtalk.org (www.healthtalk.org/peoples-experiences/mentalhealth/self-harm-parents-experiences/topics) ${ }^{43}$ may be helpful in improving parents' and carers' understanding of self harm. It is also important for those closest to the affected young person to be aware that in many cases self harm is self limiting and does not continue into adulthood. ${ }^{1}$

Mortality risks following self harm

In our study, children and adolescents who had harmed themselves were over nine times more likely to die unnaturally during the follow-up period than their unaffected peers; specifically, they were 17 times more likely to die by suicide. Although self harm is considered a lower risk behaviour in young children, ${ }^{44}$ the link between self harm and suicide is well established. It can be difficult to compare mortality risk estimates between studies owing to varying case definition and ascertainment procedures, as well as diversity in healthcare settings, periods of study, reference populations, and age categorisations. The standard WHO child and adolescent age range that we examined has not been reported on widely in relation to self harm risk. However, in a hospital based cohort study of 15-24 year olds, rates of all cause mortality and "probable suicide" were four and 10 times higher than expected, respectively. ${ }^{4}$ Among the children and adolescents who had harmed themselves in our study, $21.5 \%$ had a repeat self harm episode within 12 months, compared with hospital attendance based data, in which frequency of repetition was $17.7 \% .^{44}$ This difference further emphasises the opportunity for earlier intervention in primary care to reduce suicide risk.

\section{Strengths and limitations of this study}

In this study we examined the frequency of self harm, temporal trends, clinical management, and mortality risk from natural and unnatural causes of death in a nationally representative cohort ${ }^{16}$ of children and adolescents in primary care. Although we utilised the CPRD, one of the world's largest longitudinal primary healthcare datasets, the study did have some limitations. Firstly, accuracy of clinician Read code usage by doctors is a potential source of misclassification in primary care databases. For example, it was not possible to examine referral to private or voluntary sector services with a reasonable degree of accuracy, since such codes may be used sporadically by some doctors, and not at all by others. However, we would expect such referrals to be relatively uncommon in the healthcare setting of the NHS. ${ }^{45}$ We described the incidence and clinical management of self harm from a primary care perspective. However, this was not a complete community sample of young people who harmed themselves. An illustration of this limitation is that self cutting was less often recorded in our study and self poisoning episodes predominated, whereas the opposite has been found in community surveys. ${ }^{46}$ However, when examining the linked mortality data in the third analytical phase, we identified an additional $50 \%$ of episodes of children and adolescent self harm presenting at general practice consultations that would not have been recorded from inpatient hospital data alone-a major strength of utilising the CPRD and its linkage scheme. Secondly, studies investigating suicide tend to underestimate numbers since some coroners may be reluctant to return a suicide verdict where intent is difficult to establish, especially in younger people. Thus, we conducted a sensitivity analysis, alternately estimating hazard ratios according to two varying case definitions for suicide: suicide verdict only versus suicide verdict plus open verdicts. We found no material difference between the two relative risk estimates.

\section{Conclusion}

This large cohort study provides a unique primary care perspective on self harm among children and adolescents. We found a noticeable increase in recorded self harm episodes among girls in their 
early-mid teens, and at younger ages than previously reported. The recent sharp increase seems to be specific to 13-16 year old girls. This marked apparent increase prompts the urgent need to identify the causes of this phenomenon. Our findings highlight the important role of primary care for early intervention and inquiry, monitoring, and targeting of children and adolescents who may not openly engage with healthcare services for their current self harming behaviour. Further development of appropriate interventions is needed as there is little evidence of a consistent clinical management approach for self harm among children and adolescents, especially in primary care settings. The increased risks of all cause and cause specific mortality observed emphasise the urgent need for integrated care involving families, schools, and healthcare provision to enhance safety among these distressed young people in the short term, and to help secure their future mental health and wellbeing.

\section{AUTHOR AFFILIATIONS}

${ }^{1}$ Centre for Pharmacoepidemiology and Drug Safety, NIHR Greater Manchester Patient Safety Translational Research Centre, Manchester Academic Health Science Centre, Division of Pharmacy and Optometry, University of Manchester, Manchester, UK

${ }^{2}$ Centre for Mental Health \& Risk, NIHR Greater Manchester Patient Safety Translational Research Centre, Manchester Academic Health Science Centre, Division of Psychology and Mental Health, University of Manchester, Manchester, UK

${ }^{3}$ NIHR School for Primary Care Research, Centre for Health Informatics, Manchester Academic Health Science Centre (MAHSC), Division of Informatics, Imaging and Data sciences, University of Manchester, Manchester, UK

${ }^{4}$ Manchester Academic Health Science Centre, Division of Neuroscience and Experimental Psychology, University of Manchester, Manchester, UK; Royal Manchester Children's Hospital, Central Manchester University Hospitals NHS Foundation Trust, Manchester, UK

${ }^{5}$ Research Institute for Primary Care and Health Sciences, West Midlands Collaboration for Leadership in Applied Health Research and Care, Keele University, Staffordshire, UK

${ }^{6}$ Centre for Suicide Prevention, NIHR Greater Manchester Patient Safety Translational Research Centre, Manchester Academic Health Science Centre, Division of Psychology and Mental Health, University of Manchester, Manchester, UK; Greater Manchester Mental Health NHS Foundation Trust, Prestwich Hospital, Prestwich, Manchester, UK

Contributors: CM completed the literature search. CM, DMA, MJC, and RTW designed the study and data analysis plan. CM performed the data analysis. CM, DMA, EK, JG, MJC, NK, and RTW interpreted the results. CAC-G and NK agreed on the final clinical Read code lists. All authors critically reviewed the manuscript and approved the final version. $\mathrm{CM}$ acts as the guarantor.

Funding: This study was funded by the National Institute for Health Research (NIHR) through the Greater Manchester Primary Care Patient Safety Translational Research Centre (grant No GMPSTRC-2012-1). The views expressed are those of the authors and not necessarily those of the NHS, the NIHR, or the Department of Health. The funders of the study had no role in study design or in data collection, analysis, or interpretation.

Competing interests: All authors have completed the ICMJE uniform disclosure form at www.icmje.org/coi_disclosure.pdf. NK chaired the NICE self harm guidelines, quality standard topic expert group; is current chair of NICE adult depression guidelines, treatment and management committee, topic expert NICE suicide prevention guidelines, and member of the Department of Health Suicide Prevention Strategy Advisory Group. Views expressed in the paper are those of the authors and not those of NICE or Department of Health. The authors have no other relationships or activities that could appear to have influenced the submitted work
Ethical approval: This study is based on data from the Clinical Practice Research Datalink obtained under licence from the UK Medicines and Healthcare products Regulatory Agency. The study was approved by the independent scientific advisory committee for Clinical Practice Research Datalink research (protocol No 13_122ARA)

Data sharing: Read codes used are published on Clinicalcodes.org Electronic health records are, by definition, considered "sensitive" data in the UK by the Data Protection Act and cannot be shared via public deposition because of information governance restriction in place to protect patient confidentiality. Access to data are available only once approval has been obtained through the individual constituent entities controlling access to the data. The primary care data can be requested via application to the Clinical Practice Research Datalink (www.cprd.com/researcher), secondary care data can be requested via application to the hospital episode statistics from the UK Health and Social Care Information Centre (www.hscic.gov.uk/ hesdata), and mortality data are available by application to the UK Office for National Statistics (www.ons.gov.uk/ons/index.html).

Transparency: The lead author (CM) affirms that the manuscript is an honest, accurate, and transparent account of the study being reported; that no important aspects of the study have been omitted; and that any discrepancies from the study as planned have been explained. The corresponding author had full access to all the study data and final responsibility for publication submission.

This is an Open Access article distributed in accordance with the Creative Commons Attribution Non Commercial (CC BY-NC 4.0) license, which permits others to distribute, remix, adapt, build upon this work non-commercially, and license their derivative works on different terms, provided the original work is properly cited and the use is noncommercial. See: http://creativecommons.org/licenses/by-nc/4.0/

1 Moran P, Coffey C, Romaniuk H, et al. The natural history of selfharm from adolescence to young adulthood: a population-based cohort study. Lancet 2012;379:236-43. doi:10.1016/S01406736(11)61141-0

2 Mars B, Heron J, Crane C, et al. Differences in risk factors for self-harm with and without suicidal intent: findings from the ALSPAC cohort. Affect Disord 2014;168:407-14. doi:10.1016/j.jad.2014.07.009

3 Patton GC, Coffey C, Romaniuk H, et al. The prognosis of common mental disorders in adolescents: a 14-year prospective cohort study. Lancet 2014;383:1404-11. doi:10.1016/S01406736(13)62116-9

4 Hawton K, Harriss L. Deliberate self-harm in young people: characteristics and subsequent mortality in a 20-year cohort of patients presenting to hospital. J Clin Psychiatry 2007;68:1574-83. doi:10.4088/JCP.v68n1017

5 Patton GC, Coffey C, Sawyer SM, et al. Global patterns of mortality in young people: a systematic analysis of population health data. Lancet 2009;374:881-92. doi:10.1016/S0140-6736(09)60741-8

6 World Health Organisation. Preventing suicide: a global imperative. 2014. Geneva, Switzerland, WHO. www.who.int/mental health/ suicide-prevention/world_report_2014/en/ (Accessed 26/04/2017)

7 Office of National Statistics. Statistical bulletin: Suicides in the UK: 2015 registrations. 2016. www.ons.gov.uk/ peoplepopulationandcommunity/birthsdeathsandmarriages/deaths/ bulletins/suicidesintheunitedkingdom/2015 registrations (Accessed 26/04/2017)

8 Rodway C, Tham SG, Ibrahim S, et al. Suicide in children and young people in England: a consecutive case series. Lancet Psychiatry 2016;3:

751-9 doi:10.1016/S2215-0366(16)30094-3

9 Association of School and College Leaders and National Children's Bureau. Keeping Young People in Mind - Findings from a Survey of Schools Across England. 2016. www.ascl.org.uk/utilities/documentsummary.html?id=D91C5B0A-72A6-4117-96A9B343E51FB296 (Accessed 26/04/2017)

10 Childline and NSPCC. It turned out someone did care: Childline Annual Review 2015/16. 2016. www.nspcc.org.uk/services-andresources/research-and-resources/2016/childline-annual-review2015-16-turned-out-someone-did-care/ (Accessed 26/04/2017)

11 Department of Health. Preventing suicide in England: Third progress report of the cross-government outcomes strategy to save lives. 2017. www.gov.uk/government/uploads/system/uploads/ attachment data/file/582117/Suicide_report 2016 A.pdf (Accessed 26/04/2017)

12 Ystgaard M, Arensman E, Hawton K, et al. Deliberate self-harm in adolescents: comparison between those who receive help following self-harm and those who do not. J Adolesc 2009;32:875-91. doi:10.1016/j.adolescence.2008.10.010

13 Hawton K, Bergen H, Waters K, et al. Epidemiology and nature of selfharm in children and adolescents: findings from the multicentre study of self-harm in England. Eur Child Adolesc Psychiatry 2012;21:36977. doi:10.1007/s00787-012-0269-6 
14 Cooper J, Steeg S, Bennewith O, et al. Are hospital services for self-harm getting better? An observational study examining management, service provision and temporal trends in England. BM Open 2013;3:e003444. doi:10.1136/bmjopen-2013-003444

15 Clements C, Turnbull P, Hawton K, et al. Rates of self-harm presenting to general hospitals: a comparison of data from the Multicentre Study of Self-Harm in England and Hospital Episode Statistics. BMJ Open 2016;6:e009749. doi:10.1136/bmjopen-2015-009749.

16 Herrett E, Gallagher AM, Bhaskaran K, et al. Data Resource Profile: Clinical Practice Research Datalink (CPRD). Int Epidemiol 2015;44:827-36. doi:10.1093/ije/dyv098

17 NHS Digital. Linked HES-ONS mortality data. 2016. http://content. digital.nhs.uk/article/2677/Linked-HES-ONS-mortality-data (Accessed 26/04/2017)

18 National Institute for Health and Care Excellence. Self-harm in over $8 \mathrm{~s}$ : short-term management and prevention of recurrence. NICE Clinical guideline CG16. 2004. www.nice.org.uk/guidance/cg16 (Accessed 26/04/2017)

19 Carr MJ, Ashcroft DM, Kontopantelis E, et al. The epidemiology of self-harm in a UK-wide primary care patient cohort, 2001-2013. BMC Psychiatry 2016;16:53. doi:10.1186/s12888-016-0753-5.

20 Thomas KH, Davies N, Metcalfe C, Windmeijer F, Martin RM, Gunnell D. Validation of suicide and self-harm records in the Clinical Practice Research Datalink. Br J Clin Pharmacol 2013;76:145-57. doi:10.1111/bcp.12059

21 World Health Organization (WHO). Manual of the International Classification of Diseases, 10th Revision (ICD-10). WHO, 1992.

22 Schoenfeld D. Partial residuals for the proportional hazards regression model. Biometrika 1982;69:239-41doi:10.1093/ biomet/69.1.239.

23 Springate DA, Kontopantelis E, Ashcroft DM, et al. ClinicalCodes: an online clinical codes repository to improve the validity and reproducibility of research using electronic medical records. PLoS One 2014;9:e99825. doi:10.1371/journal.pone.0099825.

24 Herbert A, González-Izquierdo A, McGhee J, Li L, Gilbert R. Timetrends in rates of hospital admission of adolescents for violent, self-inflicted or drug/alcohol-related injury in England and Scotland, 2005-11: population-based analysis. J Public Health (Oxf) 2017;39:65-73.doi:10.1093/pubmed/fdw020

25 Tyrrell EG, Orton E, Tata LJ, et al. Changes in poisonings among adolescents in the UK between 1992 and 2012: a population based cohort study. Inj Prev 2016;22:400-6. doi:10.1136/ injuryprev-2015-041901.

26 Geulayov G, Kapur N, Turnbull P. Epidemiology and trends in non-fatal self-harm in three centres in England, 2000-2012: findings from the Multicentre Study of Self-harm in England. BMJ Open 2016;6:e010538. doi:10.1136/bmjopen-2015-010538.

27 Morthorst B, Soegaard B, Nordentoft M, Erlangsen A. Incidence Rates of Deliberate Self-Harm in Denmark 1994-2011. Crisis 2016;37:256-64. doi:10.1027/0227-5910/a000391

28 McManus S, Hassiotis A, Jenkins R, Dennis M, Aznar C, Appleby L. Chapter 12: suicidal thoughts, suicide attempts and self-harm. In: McManus S, Bebbington P, Jenkins R, Brugha T, editors. Mental health and wellbeing in England: Adult Psychiatric Morbidity Survey 2014. Leeds: NHS Digital; 2016.

29 Lessof C, Ross A, Brind R, Bell E, Newton S. Longitudinal Study of Young People in England Cohort 2: Health and Wellbeing Wave 2 Report. 2016. www.gov.uk/government/uploads/system/uploads/ attachment_data/file/540563/LSYPE2_w2_research_report.pdf (Accessed 26/04/2017)

30 Patton GC, Hemphill SA, Beyers JM, et al. Pubertal stage and deliberate self-harm in adolescents. I Am Acad Child Adolesc Psychiatry 2007;46:508-14. doi:10.1097/chi.0b013e31803065c7
31 Livingstone S, Mascheroni G, Ólafsson K, Hadon L. Children's online risks and opportunities: comparative findings from EU Kids Online and Net Children Go Mobile. 2014. www.eukidsonline.net (Accessed 26/04/2017)

32 Daine K, Hawton K, Singaravelu V, Stewart A, Simkin S, Montgomery P. The power of the web: a systematic review of studies of the influence of the internet on self-harm and suicide in young people. PLOS One 2013;8:e77555. doi:10.1371/journal.pone.0077555

33 The Children's Society. The Good Childhood Report 2016. 2016. London. www.childrenssociety.org.uk/sites/default/files/pcr090_ mainreport_web.pdf (Accessed 26/04/2017)

34 Rees G, Main G. Children's views on their lives and well-being in 15 countries: An initial report on the Children's World Survey, 2013/14 2015. York, UK: Children's World Project (ISCWeB). www.isciweb. org/_Uploads/dbsAttachedFiles/ChildrensWorlds2015-FullReportFinal.pdf (Accessed 26/04/2017)

35 Sarginson J, Webb RT, Stocks SJ, Esmail A, Garg S, Ashcroft DM Temporal trends in antidepressant prescribing to children in UK primary care, 2000-2015. J Affect Disord 2017;210:312-8. doi:10.1016/j.jad.2016.12.047

36 O'Brien D, Harvey K, Howse J, Reardon T, Creswell C. Barriers to managing child and adolescent mental health problems: a systematic review of primary care practitioners' perceptions. Br J Gen Pract 2016;66:e693-707. doi:10.3399/bjgp16X687061.

37 Abdinasir K, Pona I. Access Denied: A teenager's pathway through the mental health system. 2015. www.childrenssociety.org.uk/sites/ default/files/AccessDenied_final.pdf (Accessed 24/08/2017)

38 Hart JT. The inverse care law. Lancet 1971;1:405-12.doi:10.1016/ S0140-6736(71)92410-X

39 Hawton K, Witt KG, Taylor Salisbury TL, et al. Interventions for self-harm in children and adolescents. Cochrane Database Syst Rev 2015;(12):CD012013. doi:10.1002/14651858.CD012189.

40 National Institute for Health and Clinical Excellence. Depression in children and young people: identification and management. 2015. www.nice.org.uk/guidance/CG28 (Accessed 24/08/2017)

41 Erlangsen A, Lind BD, Stuart EA, et al. Short-term and long-term effects of psychosocial therapy for people after deliberate self-harm: a register-based, nationwide multicentre study using propensity score matching. Lancet Psychiatry 2015;2:49-58. doi:10.1016 S2215-0366(14)00083-2

42 Wasserman D, Hoven CW, Wasserman C, et al. School-based suicide prevention programmes: the SEYLE cluster-randomised, controlled trial. Lancet 2015;385:1536-44. doi:10.1016/S0140 6736(14)61213-7

43 healthtalk.org. Self-harm:Parents' experiences. 2016. www. healthtalk.org/peoples-experiences/mental-health/self-harmparents-experiences/topics (Accessed 24/08/2017)

44 Hawton K, Harriss L. Deliberate self-harm by under-15-yearolds: characteristics, trends and outcome. / Child Psychol Psychiatry 2008;49:441-8. doi:10.1111/j.1469-7610.2007. 01852.x

45 Raleigh VS, Polato GM, Bremner SA, Dhillon S, Deery A. Inpatient mental healthcare in England and Wales: patterns in NHS and independent healthcare providers. J R Soc Med 2008;101:544-51. doi:10.1258/jrsm.2008.080223.

46 Madge N, Hewitt A, Hawton K, et al. Deliberate self-harm within an international community sample of young people: comparative findings from the Child \& Adolescent Self-harm in Europe (CASE) Study. I Child Psychol Psychiatry 2008:49:667-77.

Supplementary information: Table showing proportion of children and adolescents with a comorbidity ever recorded over follow-up who self harmed 